\title{
Modeling dynamics of pressure fluctuations in a microchannel heat exchanger covered with a wire mesh membrane
}

\author{
Jerry Czarnecki ${ }^{1}$, Romuald Mosdorf ${ }^{1 *}$, Hubert Grzybowski ${ }^{1}$, Pawet Dzienis ${ }^{1}$ \\ ${ }^{1}$ Department of Mechanics and Applied Computer Science, Faculty of Mechanical Engineering Bialystok University of Technology, \\ Bialystok, Poland
}

\begin{abstract}
Pressure and mass flow fluctuations in a system consisting of a microchannel two-phase heat exchanger with wire mesh covering and overflow chamber were modelled by employing mass, momentum, and energy balances on the fluid in mesh volume. These fluctuations are a result of the alternate wetting and drying of the mesh membrane driven by a combination of evaporation and capillary pressure-driven flow into the mesh. Experiments using a system of eleven parallel microchannels, each of dimension $32 \times 0.25 \times 0.5 \mathrm{~mm}$, slotted into a copper substrate and covered with a stainless steel mesh of $0.025 \mathrm{~mm}$ wire and $0.026 \mathrm{~mm}$ aperture, confirm the occurrence of these high frequency pressure and mass flow fluctuations. The use of non-linear methods that included attractor reconstruction and recurrence plotting to enhance predictive capabilities is also explored.
\end{abstract}

* Corresponding author: r.mosdorf@pb.edu.pl 


\section{1 introduction}

In microscale channels, boiling is less stable than in normal-sized channels $[1,18]$. Nevertheless, understanding the dynamics of boiling in small channels is important for applications ranging from heat transfer in electronics and supercomputer design. Most of the studies concerning the boiling inside small diameter channels refer to two-phase flow instabilities [2], critical heat flux [3], heat transfer coefficient $[4,5]$, and pressure drop [6]. During flow boiling instabilities, various flow patterns were observed: dispersed bubbles, confined bubbles, slugs, bubbly, churn or annular [7-9]. Numerous experiments deal with visualization of two-phase flow patterns during flow boiling inside small diameter channels. Chinnov et al. [10] reported that formation of various two-phase flow patterns and transitions between them are determined by instabilities of the two-phase flow. Cheng et al. proposed a detailed two-phase flow pattern map and two-phase flow frictional pressure drop model for $\mathrm{CO}_{2}[11,12]$. Flow pattern maps were also proposed by $\mathrm{Fu}$ et al. [13], they were constructed using coordinates of a non-dimensional parameter space (boiling number, Weber number). Thome et al. [14] presented a detailed discussion on the development of two-phase flow regime maps for microchannels; they emphasized the need for creating two-phase flow maps containing physical information on the dynamic characteristics of the flow.

Two-phase flow instabilities in mini-channels cause periodic or non-periodic oscillations in the pressure drop and channel wall temperature. Static and dynamic instability are defined in the literature [15]. In the dynamic type, the system continuously transitions between different equilibrium states. In many cases, the systems are affected by several instabilities at once [2]. The oscillations caused by one type of instability are superimposed onto another type, and this superposition leads to a continuous transition between the different types of flow patterns [16]; therefore, it is difficult to distinguish a specific flow pattern sequence when dynamic instabilities are present.

In Wang, et. al [17], micromembrane-enhanced evaporating surfaces were analysed during the capillary evaporation. There it was shown that the micromembrane increases the $\mathrm{CHF}$ because of the separation of the vapour and fluid transport processes. It has been reported that oscillating flows induced by the bubble growth and collapse as well as the capillary flows induced by the receding menisci were observed and play important roles in enhancing the heat transfer.

In the present work, the stability of micromembrane-enhanced boiling in mini-channels system will be analysed.

\section{Experimental setup}

In the experimental setup shown in Fig. 1, distilled water was pumped by means of a gear pump to the surge tank. The water flow rate was measured by the mass flow meter, and the flow channel was heated electrically. The temperatures were measured using six thermocouples. The pressures at the inlet, outlet, and of the air over the mesh were measured using pressure sensors of the type MPX5050DP. Overall, the heat exchanger consisted of 11 parallel mini-channels each of dimension $32 \times 0.25 \times 0.5 \mathrm{~mm}$.

a)
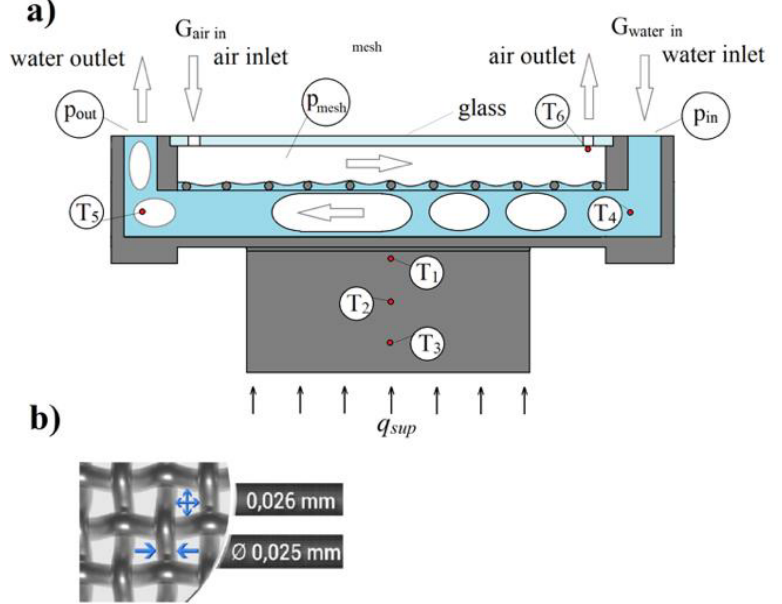

Fig. 1. Experimental setup. a) Schematic of the experimental setup: pin - inlet pressure sensor, pout - outlet pressure sensor, mesh - air pressure sensor over the mesh; Temperatures T1, T2, T3, T4, T5, T6, are measured using thermocouples (type $\mathrm{K}$ ), b) mesh dimensions.

\section{Analysis and modelling}

Examples of inlet pressure fluctuations and air pressure fluctuations over the mesh for three different flow conditions are shown in Fig. 2.

Results indicate that the inlet pressure of the channel fluid fluctuates at a low frequency in sync with the pressure fluctuations of the air but with a high frequency fluctuation superposed. This higher frequency fluctuation occurs because of the presence of the mesh and liquid evaporation within it. A model of liquid flow through the mesh is shown in Fig.3.

It is postulated that the pressure fluctuations seen in the exiting air over the mesh are caused by the following dynamics of liquid flow through the mesh and concurrent pressure fluctuations therein. One can assume that initially, the mesh is filled by liquid water from capillary forces that drive the water into the mesh, and the heat flux applied at the channel bottom causes the temperature of the fluid in the channel to rise above that in the mesh void.

A temperature gradient, $\Delta T$ (Fig.3) exists between the fluid in the microchannel and that in the mesh volume. This temperature gradient is accompanied by a liquid density gradient, $\Delta \rho$, the result of which is a flow of hot fluid in the channel into the mesh volume. The mass flow rate increases. This process is shown schematically in Fig. 3.

The flow of hot fluid into the mesh volume causes the temperature there to increase and the temperature gradient to decrease or even disappear. This increase in temperature in the mesh volume is accompanied by vapor generation, and the disappearance of the temperature gradient results in a decrease in mass flow into the volume. The evaporation process (the 
evaporative heat flux) in the mesh volume (Fig.3) causes the mesh volume to cool, and the original temperature gradient is re-established. The process of liquid flow in the mesh volume repeats.
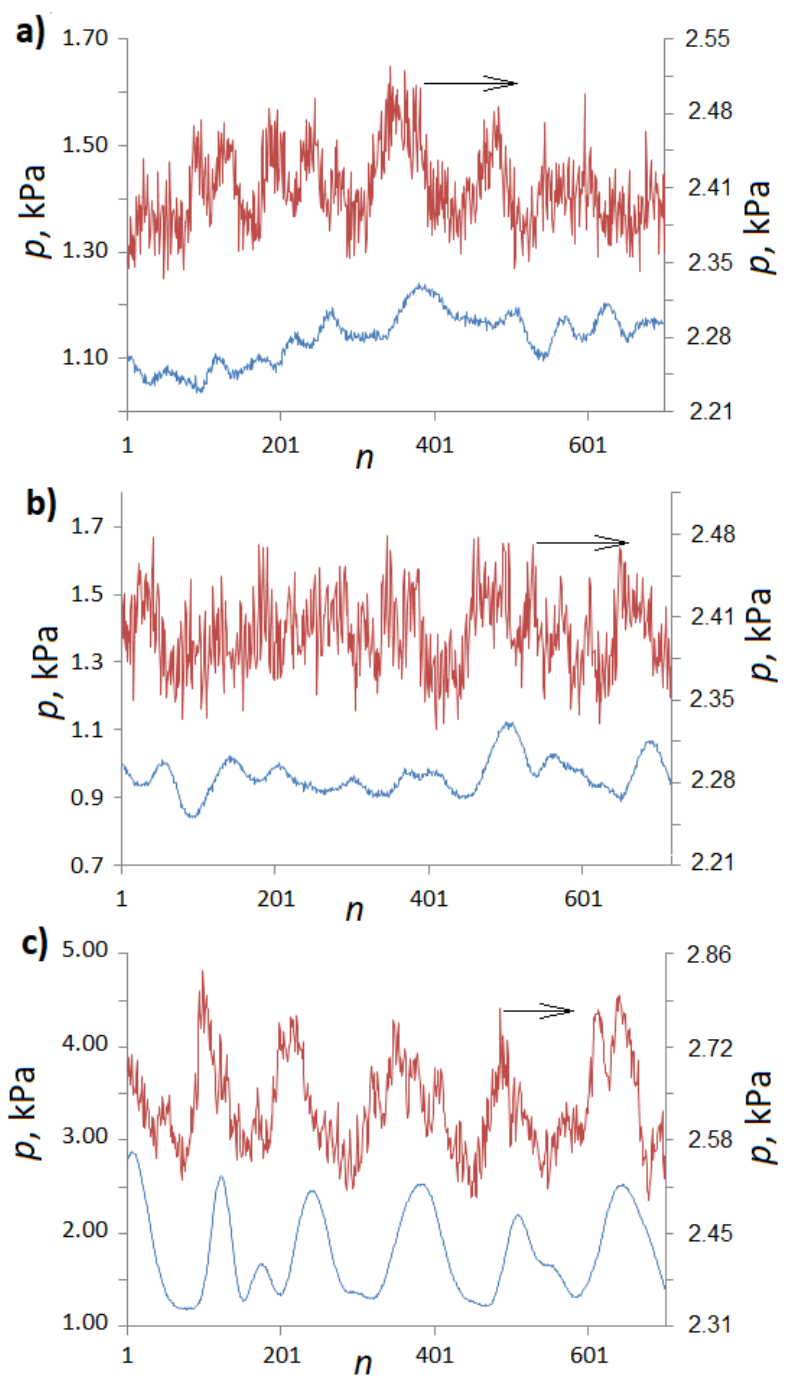

Fig. 2. Pressure fluctuations of the water at the inlet (blue) and of the air over the mesh (red) for the following three different flow conditions: a) outlet air temperature of $55^{\circ} \mathrm{C}$, $\mathrm{P}=71.4 \mathrm{~W}, \mathrm{G}=1161 \mathrm{~g} / \mathrm{h}, \mathrm{b}$ ) outlet air temperature of $67^{\circ} \mathrm{C}$, $\mathrm{P}=71.4 \mathrm{~W}, \mathrm{G}=690 \mathrm{~g} / \mathrm{h}$, and c) outlet air temperature of $94^{\circ} \mathrm{C}, \mathrm{P}=218 \mathrm{~W}, \mathrm{G}=691 \mathrm{~g} / \mathrm{h}$.

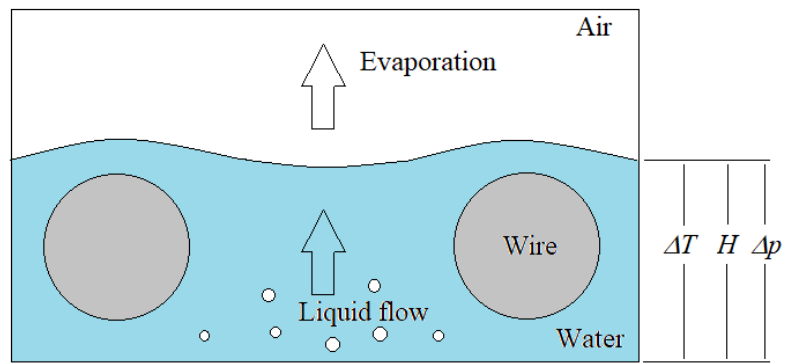

Fig. 3. The model of liquid flow through mesh aperture.

In short, cyclical temperature gradients within and countercyclical mass flow rates through the mesh are presumed to cause the observed pressure fluctuations in the liquid and cause similar fluctuations in the air above the mesh.
Performing both mass and momentum balances between the microchannel inlet and outlet in Fig. 1, it can be shown that the pressure difference between these locations is caused primarily by the mass flow from the channel into the mesh volume overhead.

A second momentum balance between the bottom and top of the mesh, of thickness $H$, is performed to establish the pressure difference between these two locations, $\Delta p$ (Fig.3). For simplicity, the mesh is modeled as a single opening above the flow microchannel through which mass is pulled by capillary action. Mass conservation dictates that the flow from the microchannel into the mesh volume equals the mass flow out of the mesh volume. Furthermore, the difference in densities between the fluid entering and leaving the mesh volume differ by $\Delta \rho$. Using this fact and noting that the pressure at the mesh bottom can be related to that at the channel inlet using Bernoulli's principle, and $\Delta p$ can be obtained as:

$$
\Delta p(H)=\frac{\dot{m}^{2}}{\rho_{0} A^{2}}\left[\frac{1}{2}+\left(\frac{1}{1-\frac{\Delta \rho}{\rho_{0}}}-1\right)\right]-g \Delta \rho H
$$

where $\dot{m}$ is the mass flow rate of fluid through the mesh, $\rho_{0}$ is the density of the fluid at the bottom of the mesh under conditions of no mass flow, i.e. $\dot{m}=0$, and $A$ is the effective cross-sectional area of the mesh volume through which the mass of fluid flows.

It is important to note that this area does not necessarily equal the total cross-sectional area of the mesh volume but is reduced due to effects that cause the fluid directly adjacent to the mesh wire to stagnate, likely boundary and capillary effects. Therefore, in the model used here, the cross-sectional area of the single opening does not, in general, equal that of the mesh but is reduced due to the aforementioned effects.

For small mass flow rates, the difference between the pressure at the top of the mesh and the pressure that would exist there without any mass flow is small and can be neglected. The following expressions for $\dot{m}$ are then obtained:

$$
\dot{m}=\rho_{0} A \sqrt{g H} \sqrt{\frac{\Delta \rho / \rho_{0}}{\left(\frac{1}{1-\frac{\Delta \rho}{1^{\prime}} / \rho_{0}}-1\right)+\frac{1}{2}}}
$$

or:

$$
\dot{m}=\rho_{0} A \sqrt{g H} \sqrt{\frac{\beta \Delta T}{\left(\frac{1}{1-\beta \Delta T}-1\right)+\frac{1}{2}}}
$$

where it is noted that, for liquids, density changes are functions of the thermal coefficient of expansion, $\beta$ and temperature change.

In Equations $2 \mathrm{a}$ and $2 \mathrm{~b}, H$ is dependent on temperature; in fact, the height to which the fluid rises in the mesh void is a function of the capillary forces that exist between water and the mesh material as well as the density-dependent hydrostatic pressure there. Equations $2 \mathrm{a}$ and $2 \mathrm{~b}$ establish the mass flow rate as a function of the density change, $\Delta \rho$ or $\Delta T$. 
If one assumes that in a given time period, $n$, liquid is transferred through the mesh volume whereby the mass flux of this transfer, $\dot{m}_{n}$, is described by Eq.(2a), and in the next time period, $n+1$, this liquid evaporates, whereby its mass flux is denoted as $\dot{m}_{n+1}$, then one can convert Eq.2 to a discrete iteration map of the following form:

$$
x_{n+1}=r \sqrt{\frac{x_{n}}{\left(\frac{1}{1-x_{n}}-1\right)+\frac{1}{2}}}
$$

where: $x=\Delta \rho / \rho_{0}, \quad r=E \rho_{0} A \sqrt{g H}$, and $E$ is a coefficient that accounts for the effects of evaporation within the mesh on fluid density. Successive mass flow rates are related by: $\dot{m}_{k+1}=E \dot{m}_{k}$.
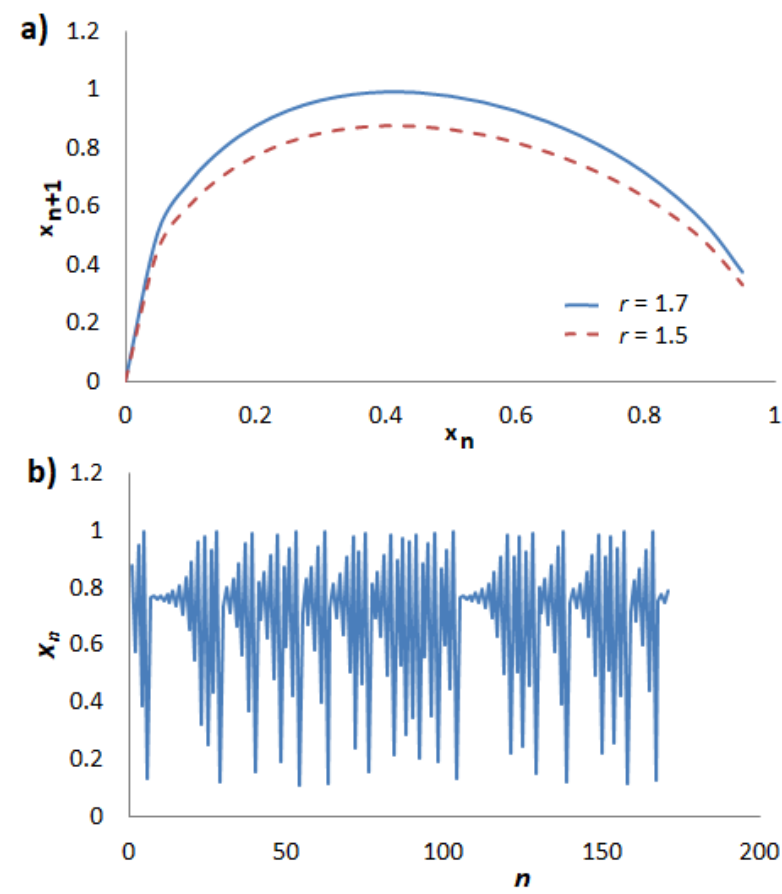

Fig. 4. Properties of Eq.3. a) graph of Eq.3 for different values of the coefficient $r, b$ ) example of an iteration of Eq. 3, for $\mathrm{r}=1.7$ and a staring value of $\mathrm{x} 0=0.2$.

The shape of the curve in Fig.4a suggests that percentage density changes about approximately 0.5 will cause the mass flow rate to behave erratically. Below about 0.5 , increasing density changes will cause the mass flow rate to increase, and above about 0.5 , increasing density changes will cause the mass flow rate to decrease. Fluctuations around this value are responsible for the chaotic behavior observed in experiments.

The results of repeated iteration of Eq. 3 are shown in Fig.4b. Starting the iteration with a value of $x_{0}=0.2$, one notices that the $x_{i}$ vary in a chaotic fashion, much like the variation observed experimentally. In general, the value of $r$ is dependent on the liquid temperature and air pressure over the mesh.

In order to analyze the influence the liquid temperature and pressure through the mesh has on the character of air pressure fluctuations, The Cross Recurrence Plot (CRP) has been used.

A Cross Recurrence Plot $(C R P)$ is created from two time series, $x_{i}$ and $y_{j}$, which are embedded in the same dynamical phase space. Creating a single $C R P$ allows one to observe the recurrence of the state of a dynamical system consisting of $x_{i}$ and $y_{i}$ trajectories embedded in an $m$-dimensional space. The times at which a state of the dynamical system $x_{i}$ is close to the state of the dynamical system $y_{i}$ are marked in the plot. Formally, the cross recurrence plot is defined as [19, 20]:

$$
C R_{i, j}=\Theta\left(\varepsilon-\left\|x_{i}-y_{j}\right\|\right)
$$

where $\varepsilon$ is a threshold distance, \|\| is a norm in $m-$ dimensional space, and $\Theta$ is the Heaviside function.

The CRP is created based on an attractor reconstructed in phase space. The reconstruction of the attractor in a certain embedding dimension, $m$, is carried out stroboscopically. The subsequent coordinates of attractor points are as follows [20].

$$
\left\{x_{i}, x_{i+\tau}, \ldots x_{i+(m-1) \tau}\right\}
$$

where $x_{i}$ is the measured quantity, and $\tau$ is a time delay.

The image of the attractor in $m$-dimensional space depends on the time delay $-\tau$. The mutual information [21] between the time series $x_{i}$ and $x_{(i+\tau)}$ can be used to determine proper time delay for the attractor reconstruction. This mutual information of $x_{i}$ and $x_{(i+\tau)}$ time series is defined as [22]:

$I\left(x_{i}, x_{i+\tau}\right)=\sum_{x_{i+\tau}} \sum_{x_{i}} p\left(x_{i}, x_{i+\tau}\right) \log _{2}\left[\frac{p\left(x_{i}, x_{i+\tau}\right)}{p\left(x_{i}\right) p\left(x_{i+\tau}\right)}\right]$ where $p\left(x_{i}, x_{(i+\tau)}\right)$ is the joint probability distribution function of $x_{i}$ and $x_{(i+\tau)}$, and $p\left(x_{i}\right)$ and $p\left(x_{i+\tau}\right)$ are the marginal probability distribution functions of $x_{i}$ and $x_{(i+\tau)}$.

As $\tau$ is increased, the mutual information decreases and then it rises again. The time delay for which the mutual information obtains the first minimum is a proper value of $\tau$ for the attractor reconstruction [23]. The mutual information is equal to zero if the two time series are independent.

The false nearest neighbour algorithm [24] was used for the estimation of the proper embedding dimension of the attractor. In this method, the changes in the number of neighbouring points in the embedding space with increasing embedding dimension is examined. For each point $x_{i}$, the distances to its nearest neighbours $x_{j}$ are calculated in both the $m$ and $m+1$ dimensional embedding spaces. The point is treated as a false neighbour when the distance between points $(i, j)$ increases together with increasing the embedding dimension. The number of false neighbours is calculated for the whole time series and for several dimensions until the fraction of false points reaches zero. Such a dimension is treated as a proper embedding dimension for the attractor reconstruction.

The number of points, which appear in the recurrence plot, depends on the value of the threshold distance $\varepsilon$. The number of points in the recurrence plot can be quantified by the recurrence rate, RR, which is a ratio of recurrence points to all possible recurrence points in the $R P$. 
The Determinism parameter is defined as the percentage of recurrence points which lie on the diagonal line to all possible recurrence points in the $R P$. It is a measure of the deterministic character of the flow. This parameter is calculated for the given flow as are the lengths of the vertical and horizontal lines, which indicate times during which a state does not change or changes very slowly; the lengths of these lines can be considered measures of trajectory stability and synchronisation [20].

\section{Results and discussion}

Examples of the cross recurrence plots created from fluctuations of the inlet water pressure and air pressure over the mesh are shown in Fig. 6. Inlet water pressure fluctuations have been used to estimate the time delay and embedding dimension for the attractor reconstruction. The obtained results demonstrate how pressure changes above the mesh synchronize with changes in water pressure as well as with changes in the temperature achieved by the air leaving the area above the mesh.

The determinism parameter, length of longest diagonal line, and length of longest vertical line are considered here as relevant measures of similarity between two systems. One can notice in Fig. 6 that synchronisation between the inlet pressure fluctuations and outlet air pressure fluctuations increase with increasing outlet air temperature. The synchronization between these fluctuations is an effect of the uniform boiling intensity seen in all 11 flow channels when the electrically-generated heat flux is increased.

\section{Conclusions}

The dynamics of heat transfer in microchannels covered with wire mesh have been examined using both physical considerations as well as tools from the field of chaos and dynamics. Results using simplified models of the microchannel with mesh covering indicate qualitatively that the dynamics of liquid flow through the mesh may be responsible for the erratic flow and pressure fluctuations observed in experiments. Furthermore, synchronization phenomena between various parameters at different locations are explained using attractor reconstruction and recurrence plotting. Nevertheless, consideration of a more detailed wire mesh geometry and the dynamics of dissipative flow through it would provide additional insight into the physical causalities likely responsible for the observed fluctuations, and such work is planned for the future.

This work was supported by the National Science Centre, Poland UMO-2017/27/B/ST8/02905.

\section{References}

1. L. Cheng, G. Ribatski, J.R. Thome, Appl. Mech. Rev. 61 050802-28 (2008).
2. L.C. Ruspini, C.P. Marcel, A. Clausse, Int. J. Heat Mass Transf. 71 521-548 (2014).

3. P.K. Das, A.K. Das, S.K. Saha (Ed.), Microchannel Phase Change Transp. Phenom., Butterworth-Heinemann, 217-255 (2016).

4. M. Piasecka, B. Maciejewska, Exp. Therm. Fluid Sci. 68 459-467 (2015).

5. L. Cheng, S.K. Saha (Ed.), ButterworthHeinemann, 141-191 (2016).

6. M.V. Sardeshpande, P. Shastri, V.V. Ranade, Int. J. Heat Fluid Flow. 61 636-649 (2016).

7. L. Yin, L. Jia, Exp. Therm. Fluid Sci. 74 247-256 (2016).

8. M.H. Maqbool, B. Palm, R. Khodabandeh, Exp. Therm. Fluid Sci. 46 120-130 (2013).

9. X.L. Xie, Z.J. Liu, Y.L. He, W.Q. Tao, Appl. Therm. Eng. 29 64-74 (2009).

10. E.A. Chinnov, F.V. Ron'shin, O.A. Kabov, Int. J. Multiph. Flow. 80 57-68 (2016).

11. L. Cheng, G. Ribatski, J. Moreno Quibén, J.R. Thome, Int. J. Heat Mass Transf. 51 111-124 (2008).

12. L. Cheng, G. Ribatski, J.R. Thome, Int. J. Heat Mass Transf. 51 125-135 (2008).

13. B.R. Fu, P.H. Lin, M.S. Tsou, Chin Pan, International Journal of Heat and Mass Transfer, Volume 55, Issues 5-6, 1754-1763 (2012)

14. J.R. Thome, A. Bar-Cohen, R. Revellin, I. Zun, Exp. Therm. Fluid Sci. 44 1-22 (2013).

15. J.A. Boure, A.E. Bergles, L.S. Tong, Nucl. Eng. Des. 25 165-192 (1973).

16. S.M. Ghiaasiaan, Two-Phase Flow, Boiling, and Condensation: In Conventional and Miniature Systems, Cambridge University Press, (2007).

17. G. Wang, P. Cheng, H. Wu, Int. J. Heat Mass Transf. 50 4297-4310 (2007).

18. M.M. Padki, H.T. Liu, S. Kakac, Int. J. Heat Fluid Flow. 12 240-248 (1991).

19. J.-P. Eckmann, S.O. Kamphorst, D. Ruelle, EPL Europhys. Lett. 4973 (1987).

20. N. Marwan, M. Carmen Romano, M. Thiel, J. Kurths, Phys. Rep. 438 237-329 (2007).

21. M.S. Roulston, Phys. Nonlinear Phenom. 125 285-294 (1999).

22. H.G. Schuster, W. Just, Deterministic Chaos: An Introduction, John Wiley \& Sons, (2006).

23. H. Kantz, T. Schreiber, Nonlinear Time Series Analysis, Cambridge University Press, (2004).

24. N. Marwan, Cross Recurrence Plot Toolbox for MATLAB ${ }^{\circledR}$, Ver. 5.21 (R31c), http://tocsy.pikpotsdam.de/CRPtoolbox/. 